www.jmscr.igmpublication.org

Index Copernicus Value: 79.54

ISSN (e)-2347-176x ISSN (p) 2455-0450

crossref DOI: https://dx.doi.org/10.18535/jmscr/v7i4.80

\title{
Haemostatic Radiotherapy Dosing Schedules: A Review Article
}

Authors

\author{
Shashank Shenoy, Rajan Yadav, Ankita Parikh, Maitrik Mehta, U Suryanarayan \\ Department of Radiation Oncology, The Gujarat Cancer and Research Institute, Ahmedabad, Gujarat \\ Email: dr.shenoyshashank@gmail.com, Mob: +91-9900916167
}

\section{Introduction}

Approximately $10 \%$ of patients of advanced cancer present with bleeding. This can be a distressing sight alike for patient and care givers. in a country like ours, this presentation is of particular value as about $40 \%$ patients present with advanced stage. Bleeding can present in many ways including hematemesis, haematochezia, melena, hemoptysis, haematuria, epistaxis, vaginal bleeding or ulcerated skin lesions. a majority of them resolve by pharmacological measures and compression dressings, for those which don't resolve radiotherapy can be used. In such situations radiotherapy (RT) has been used with great effect. This review focuses on radiotherapy when used with haemostatic intent. Generally haemostatic RT is believed to be an effective treatment for patients with bleeding due to cancer. Relatively little published literature is available which focuses on bleeding control of haemostatic RT and even less is known about the optimal RT total dose and fractionation.

Keywords: Haemostasis, Apoptosis, Radiotherapy

\section{Methods}

In this review article we have analysed 5 studies and summarised the various dosing schedules used. We provide an updated, evidence-based narrative review of treatment of haemorrhagic of various advanced malignancies using different radiation dosing and fractionation schedules.

\section{Discussion}

There are a number of modalities conventionally used to manage haemorrhage in patients with advanced malignancy ${ }^{[1]}$. Systemic treatments include blood products, vitamin K, vasopressin or desmopressin, somatostatin analogues, and antifibrinolytic agents. Local modalities include topical haemostatic agents, dressings, endoscopy, vessel ligation, tissue resection, cauterisation, styptics, transcutaneous arterial embolization, or balloon placement. The use of RT is also increasing, as demonstrated by numerous case reports and series which have been analysed and reviewed. The exact mechanism underlying the ability of RT to control malignant bleeding is unclear. RT might work by damaging malignant blood vessels, which are more abundant in haemorrhagic tumours, such as renal cell carcinoma and melanoma. Radiation may affect the pathophysiological process of malignant vessels through inducing malignant endothelial cell damage secondary to extensive DNA damage. It may also cause the activation of signal 
transduction pathways that can lead to cell cycle arrest or apoptosis. Bleeding in tumours can also be secondary to vascular injury from local tumour invasion, or a systemic coagulopathy caused by paraneoplastic syndromes. However, RT will not stop bleeding from normal vessels that have been invaded by cancerous cells, for example, carotid blowouts in progressing head and neck malignancy.

Radiotherapy has also been shown to be highly effective in achieving haemostasis in unresectable gastric cancers. A retrospective review of 30 patients with gastric cancer bleeding who underwent palliative RT found improvement in haemoglobin levels and decreased need for blood transfusions in $91 \%$ of cases. In a recent retrospective study of 62 patients with advanced cancer, the role of haemostatic RT was evaluated in reducing malignant bleeding.

Cihoric et $a l^{[2]}$ analysed 62 patients all with advanced incurable cancer excluding one patient whose cause was benign. Patients were treated by external beam RT, high-dose rate brachytherapy, monotherapy or a combination of both. Due to the different fractionation schedules used, the dose prescriptions were converted into 3-Gy equivalent doses and a total dose of 30 Gy using 3 Gy daily fractions was used as treatment schedule. The result was statistically significant $(\mathrm{p}<0.001)$ and bleeding was improved in 54 patients (87\%); 63\% of whom had a complete response (CR) to bleeding. Of the remaining patients, bleeding was unchanged in 7 patients (11\%) and 1 patient showed progression.

Asakura et $a l^{[3]}$ reviewed the data for all patients with gastric cancer with grade 2 bleeding i.e. requiring blood transfusion treated with $\mathrm{RT}$. The purpose of this study was to evaluate the effectiveness of $30 \mathrm{~Gy}$ in 10 fractions for bleeding from advanced gastric cancer. RT was defined as effective if the patients did not require blood transfusions for 1 or more months after the beginning of treatment. 22 of 30 patients $(73 \%)$ responded to RT. Out of the 26 patients with melena or hematemesis, 20 showed an improvement in their symptoms (77\%). The median rebleeding-free survival time from the beginning of RT was 2.6 months. The median rebleeding-free survival was 1.7 months for RT alone compared with 5.5 months for CRT ( $\mathrm{P}=$ 0.002). A dose-fractionation regimen of $30 \mathrm{~Gy}$ in 10 fractions showed a high response rate of $73 \%$ for treatment of bleeding from advanced gastric cancer. This schedule appears to have reduced the treatment period relative to $40 \mathrm{~Gy}$ in 16 fractions or $35 \mathrm{~Gy}$ in 14 fractions, which have been used for a large portion of cases in previous studies.

Biswal et $a l^{[4]}$ studied twenty cases of carcinoma of the uterine cervix presenting with heavy vaginal bleeding between April 1987 and May 1992. All had initially been managed by vaginal packing, styptics and sedatives, which had failed. Each was a histologically proven case of squamous cell carcinoma. The dosage schedule for external radiotherapy ranged from 5 Gy in a single fraction to $20 \mathrm{~Gy}$ in five fractions. The age distribution of the study group varied between 30 and 60 years with a median age of 42 years. The mean duration of vaginal bleeding was recorded as 6.25 months, becoming heavy in the last $1-5$ days. Twelve cases were managed with external haemostatic radiotherapy, three with intracavitary radiotherapy, and five were treated with a combination of external and intracavitary radiotherapy. Kraiphibul et al. ${ }^{[13]}$ used three to six Gray fractions with $62.9 \%$ bleeding control within three fractions and $97 \%$ control within five fractions of radiotherapy.

Barnes et $a l^{[5]}$ used 24Gy in three fractions, delivered on days 0,7 , and 21 over 3 weeks, as their palliative RT regimen for patients with non melanoma skin cancers. This regimen of palliative RT showed impressive outcomes, with symptom palliation in $61 \%$ of their follow-up patients. However, this retrospective study was compounded by various factors including a small sample size, short follow up periods for assessment of late effects, and elderly subjects who were likely to die from other causes. 
Helena Sung-In Jang et al ${ }^{[6]}$ used a total dose of 20Gy as a standard approach for both their cases, this dose was adequate for haemostasis and has demonstrated that radiation can also cause haemostasis in bleeding skin malignancies in neoadjuvant and palliative settings. In both of these cases, malignant bleeding presented a lifethreatening event and was contained successfully by RT. From their report, it was suggested that the optimal RT total dose and fractionation for attaining haemostasis in skin cancer is $20 \mathrm{~Gy}$ in 5 fractions. This controlled malignant bleeding even in large tumours.

\section{Conclusion}

In our institute data focusing on bleeding control in advanced cancer patients strongly suggests that haemostatic RT is a very effective treatment for significantly reducing bleeding of various primary tumours and treatment sites, bleeding skin malignancies in neoadjuvant and palliative settings without major toxicity. We commonly employ a dose schedule of 12 Gy in 3 fractions in 4 Gy per fraction in daily consecutive doses.

\section{References}

1. J. Pereira and T. Phan, "Management of bleeding in patients with advanced cancer," Oncologist, vol. 9, no. 5, pp. 561570, 2004.

2. N. Cihoric, S. Crowe, S. Eychm"uller et al., "Clinically significant bleeding in incurable cancer patients: effectiveness of haemostatic radiotherapy," Radiation Oncology, vol. 7, no. 1, p. 132, 2012.

3. H. Asakura, T. Hashimoto, H. Harada et al., "Palliative radiotherapy for bleeding from advanced gastric cancer: is a schedule of $30 \mathrm{~Gy}$ in 10 fractions adequate?" Journal of Cancer Research and Clinical Oncology, vol. 137, no. 1, pp. 125-130,2011.

4. B. M. Biswal, "Hemostatic radiotherapy in carcinoma of the uterine cervix," International Journal of Gynecology and
Obstetrics, vol. 50, no. 3, pp. 281-285, 1995.

5. E. A. Barnes, D. Breen, S. Culleton et al., "Palliative radiotherapy for non-melanoma skin cancer," Clinical Oncology, vol. 22, no. 10, pp. 844-849, 2010.

6. Jang, Helena Sung-In et al. "Radiotherapy can cause haemostasis in bleeding skin malignancies." Case reports in medicine vol. 2012 (2012)

7. J. A. Langendijk, N. K. Aaronson, J. M. A. De Jong et al., "Prospective study on quality of life before and after radical radiotherapy in non-small-cell lung cancer," Journal of ClinicalOncology, vol. 19, no. 8, pp. 2123-2133, 2001.

8. J. Tey,M. F. Back, T. P. Shakespeare et al., "The role of palliative radiation therapy in symptomatic locally advanced gastric cancer," International Journal of Radiation Oncology BiologyPhysics, vol. 67, no. 2, pp. 385-388, 2007.

9. K. Hashimoto, H. Mayahara, A. Takashima et al., "Palliative radiation therapy for hemorrhage of unresectable gastric cancer: a single institute experience," Journal of Cancer Research and Clinical Oncology, vol. 135, no. 8, pp. 1117-1123, 2009.

10. J. A. Lee, D. H. Lim, W. Park, Y. C. Ahn, and S. J. Huh, "Radiation therapy for gastric cancer bleeding," Tumori, vol. 95, no. 6, pp. 726-730, 2009.

11. M. I. Koukourakis, "Tumour angiogenesis and response to radiotherapy," Anticancer Research, vol. 21, no. 6, pp. 4285-4300, 2001.

12. S. Kempin, "Disorders of hemostasis in malignancy," Current Problems in Cancer, vol. 4, no. 4, pp. 21-26, 1979

13. Kraiphibul P, Srisupundit S, Kiatgumjakajom S, Pairachvet V. The experience in using whole pelvic irradiation in management of massive bleeding from carcinoma of the uterine 
cervix. J Med Assoc Thailand 1993; 76 Suppl 1: 78-81

14. Gagnon, Bruno \& Mancini, I \& Pereira, J \&Bruera, Eduardo. (1998). Palliative management of bleeding events in advanced cancer patients [Case report]. Journal of palliative care. 14. 50-4. 